\title{
Review
}

\section{Human papillomaviruses: The cervical cancer saga in developing countries}

\author{
Fatimah Saeed Alhamlan ${ }^{1,2}$, Ali Saeed Al-Zahrani ${ }^{3}$, Shaihana Abdulrahman Almatrrouk ${ }^{1}$, Mohammed \\ Noorain Al-Ahdal ${ }^{1,2}$
}

\author{
${ }^{1}$ Department of Infection and Immunity, King Faisal Specialist Hospital and Research Center, Riyadh, Saudi Arabia \\ ${ }^{2}$ College of Medicine, Alfaisal University, Riyadh, Saudi Arabia \\ ${ }^{3}$ Gulf Centre for Cancer Control and Prevention, King Faisal Special Hospital and Research Centre, Riyadh, Saudi \\ Arabia
}

\begin{abstract}
Cervical cancer is the fourth most common cancer affecting women, with worldwide annual incidence and mortality rates of 528,000 and 266,000 , respectively, according to the World Health Organization. It is well established that cervical cancer is predominantly caused by a persistent human papillomavirus (HPV) infection of cervical cells. Increasing numbers of studies have investigated HPV and cervical cancer, contributing greatly to the global knowledge and unraveling some of the critical questions regarding HPV transmission, infection, and prevention. However, despite these studies, our knowledge is far from complete and much remains to be discovered. Although molecular detection and HPV prophylactic approaches have greatly advanced in recent years, approximately $85 \%$ of the global burden of mortality from cervical cancer still occurs in developing countries. Clinical and molecular epidemiological studies have demonstrated a need for developing countries in general to adopt cervical screening and vaccination programs. However, studies examining cervical cancer screening modalities and HPV prevalence as well as whether HPV vaccination programs should be implemented are lacking at the national level in some developing countries. Therefore, this review describes the current status of HPV in developing countries, presenting some of the existing challenges in implementing cervical screening and HPV vaccination programs.
\end{abstract}

Key words: cervical cancer; human papillomavirus; developing countries; women's health.

J Infect Dev Ctries 2017; 11(11):819-825. doi:10.3855/jidc.9704

(Received 20 August 2017 - Accepted 16 October 2017)

Copyright (C) 2017 Alhamlan et al. This is an open-access article distributed under the Creative Commons Attribution License, which permits unrestricted use, distribution, and reproduction in any medium, provided the original work is properly cited.

\section{Introduction}

Henrietta Lacks died of cervical carcinoma in October 1951 at age 31. Since then, cells from her tumors (named HeLa cells) have been maintained, and they grow steadily when guarded from contamination [1]. The HeLa cell line remains a mainstay, even now, in many biology and virology laboratories. Although HeLa cells have been frequently used to propagate various viruses for use in laboratory diagnosis and research, studies in the 1950s and 1960s showed that these cells contain segments of DNA belonging to the high-risk human papillomavirus (HPV) type 18. Subsequent studies, for which Harald zur Hausen shared the Nobel Prize in Physiology or Medicine in 2008, have shown that HPVs are the major cause of cervical cancer and are involved in other cancers [2,3].

Papillomaviruses are ubiquitous and are members of a family (Papillomaviridae) of DNA viruses that infect humans and animals. Human papillomaviruses are small non-enveloped viruses that contain a doublestranded, closed circular DNA genome, and this genome comprises approximately 8,000 base pairs with at least eight open reading frames that separate the genome into three regions. The first region, called the long control region, regulates the replication and transcription of the viral genome. The second region is called the early region (E). The E region contains six structural proteins (E1, E2, E4, E5, E6, and E7) that are involved in viral replication and oncogenesis. The third region is called the late region $(\mathrm{L})$. It encodes the $\mathrm{L} 1$ and L2 structural proteins, which form the major and minor capsid proteins, respectively [4]. Similar to all papillomaviruses, HPVs are capable of establishing productive infections only in skin keratinocytes or mucous membranes. Most currently known HPV types cause no symptoms in the vast majority of people; however, some types cause relatively mild symptoms, such as warts (verrucae), while other HPV types are less innocuous, leading to cancers of the cervix, vulva, vagina, penis, oropharynx, or anus. More than 120 subtypes of HPVs have been classified based on their oncological potential of transforming cells: the low-risk 
Table 1. High-risk and low-risk HPVs.

\begin{tabular}{lll} 
HPV class & Prevalent genotype & Human diseases \\
\hline Low risk & $6,11,40-44,53,54,61,72,73$, and 81 & $\begin{array}{l}\text { Common warts, laryngeal papillomatosis, anogenital warts, } \\
\text { and oral papilloma }\end{array}$ \\
High risk & $16,18,31,33,35,39,45,51,55,58$, and 59 & Genital cancer, anal dysplasia, and oropharyngeal cancer \\
\hline
\end{tabular}

type may cause benign hyperproliferative lesions, or genital warts, whereas the high-risk type has been strongly associated with cervical lesions (Table 1) [5].

According to the World Health Organization (WHO), 85\% of cervical cancer cases occur in developing countries, and these cases generate a worldwide burden. Their latest report indicated that the incidence of cervical cancer cases in developing countries is more than half a million, with half of these women dying from the disease [6,7]. Therefore, this review also discusses the challenges facing developing countries combating sexually transmitted infections, especially HPV because it is the major cause of cervical cancer, and attempting to establish effective cervical screening programs.

\section{HPV and cervical cancer}

As stated above, the association between HPV and cervical cancer is well established, and cervical cancer is considered one of the most common gynecological malignant neoplasms worldwide. Approximately 5\% of the worldwide cancer incidence in 2008 was attributed to HPV infections, and $85 \%$ of HPV-related cancer was associated with cervical cancer [8]. Of the approximately 630 million women with HPV infections reported globally, 190 million had clinical infections, 528,000 were newly diagnosed with cervical carcinoma, and 266,000 died (6). While cervical cancer development is a multistep process, the disease can only develop in the presence of a persistent HPV infection of the cervical epithelium. However, the presence of HPV alone is insufficient to cause cervical cancer $[9,10]$. Although every abnormal or dysplastic lesion of the cervix must be considered potentially malignant, as it may develop into cervical cancer with time, not every HPV cervical infection leads to cervical cancer. In the Papanicolaou test (commonly called a Pap test or Pap smear), cervical epithelial cells are stained to allow for microscopic detection of abnormalities. The Pap test is conducted worldwide as a primary cervical tissue screening tool and for detection of women at risk of disease progression [11]. Laboratories generally report the results of these screens based on the Bethesda System, with squamous cell abnormalities categorized (from the mildest to the most severe) as atypical squamous cells of undetermined significance, low grade squamous intraepithelial lesion, high grade squamous intraepithelial lesion, and cervical cancer [12].

Most HPV infections are asymptomatic and resolve within two years; however, in some cases, the infection persists and progresses to cervical dysplasia. An infection is generally initiated by the onset of viral replication leading to low-grade cervical intraepithelial neoplasia (CIN1). If the virus persists and the neoplastic changes remain untreated, CIN2 may develop. Persistent HPV infection could then result in progression to high-grade lesions (i.e., CIN3) and to invasive cervical carcinoma. It is not fully understood how an HPV infection triggers cervical cells to become abnormal. However, it appears that abnormal development occurs primarily when high-risk HPVs express the viral oncogenes E6 and E7. The HPV E6 and E7 early genes are viral oncoproteins that detrimentally disturb the cell cycle by altering the function of cell cycle regulators. These two primary oncoproteins inactivate two tumor suppressor proteins, p53 (inactivated by E6) and retinoblastoma (pRb, inactivated by E7), leading to cell cycle disruption and cellular transformation [13].

\section{HPV and cervical cancer in Arabian Peninsula countries}

Six countries located on the Arabian Peninsula side of the Gulf comprise the Cooperation Council for the Arab States of the Gulf, formerly known as the Gulf Cooperation Council (GCC). These countries and their estimated current population (in millions) are Bahrain (1.4), Kuwait (3.4), Oman (3.7), Qatar (2.2), Saudi Arabia (29.1) and the United Arab Emirates (UAE) (9.4). Unfortunately, fewer than 100 articles examining HPV and cervical cancer in GCC countries have been published. However, cervical cancer ranked seventh $(2.9 \%)$ of all cancers for women in the GCC countries over a ten year period (from 1998 to 2012), according to the Gulf Center for Cancer Control and Prevention (GCCCP) registry [14]. Still, the total number of cervical cancer cases registered in GCC countries (Table 2) is the lowest in the world, and this may be attributed to differences in sexual practice, lifestyle, and genetic predisposition. Despite the lower number of cases, what is alarming is that most women with 
Table 2. Number of cervical cancer cases by GCC country from 1998 to 2012 according to the Gulf Center for Cancer Control and Prevention registry.

\begin{tabular}{cccc}
\hline GCC Countries & $\begin{array}{c}\text { Total number of cervical } \\
\text { cancer cases }\end{array}$ & $\begin{array}{c}\text { Percent of all reported } \\
\text { cervical cancer cases }\end{array}$ & ASR* \\
\hline UAE & 185 & 5.7 & 6.0 \\
Bahrain & 128 & 3.4 & 6.1 \\
KSA & 1418 & 2.2 & 1.9 \\
Oman & 398 & 5.7 & 7.0 \\
Qatar & 52 & 3.4 & 6.6 \\
Kuwait & 173 & 2.5 & 3.7 \\
\hline
\end{tabular}

*Age-standardized rate, annual rate per 100,000 persons at risk.

cervical cancer in GCC countries do not seek treatment until later stages of the disease. Indeed, almost half of all patients arrived at the hospital with regional or distant metastasis stages, decreasing the survival rate. As shown in Figure 1, over a 14-year span, only $28 \%$ of patients in GCC countries sought medical care for early-stage cervical cancer. This is especially unfortunate because cervical cancer is the most preventable cancer and has a high survival rate when detected in early stages [15]. Such data highlight the dire need for implementing cervical screening programs and awareness campaigns to educate girls, women, and healthcare providers about the importance of early detection.

The GCC countries have licensed the use of HPV vaccines, and the UAE was the first country in the European, Middle Eastern, and north African regions to implement an HPV vaccination program (in Abu Dhabi) and to introduce a voluntary school-based vaccination program for girls aged 15-17 years [16]. However, because cervical cancer is not among the most common cancers in the GCC region, the costeffectiveness of implementing HPV vaccination programs is debatable. Further evaluation will be required, especially given the current changes in socioeconomic status, lifestyles, and reproductive patterns of individuals in the GCC region. For example, marriage in some areas includes the practice not only of polygyny but also of temporary marriage, such as mesiar and mesfar, potentially increasing the prevalence of HPV infection in regions that are considered to be conservative communities.

Recent studies using polymerase chain reaction (PCR) analysis conducted in Saudi Arabia, the largest in size and population of the GCC countries, show a relatively high prevalence of HPV infection [17-19]. One study investigated HPV 16/18 DNA prevalence among women attending a family medicine clinic for routine gynecological examinations in Riyadh, the capital of Saudi Arabia. In total, 120 cervical swab specimens were collected and analyzed using PCR followed by Southern blotting [20]. The overall prevalence of HPV $16 / 18$ was $31.6 \%$. The prevalence for HPV 16 alone was $13.3 \%$ and for a mixed HPV 16 and HPV 18 infection it was $15 \%$, indicating that $28.3 \%$ of the specimens harbored HPV 16 infection. The prevalence of HPV 18 alone was $3.3 \%$, indicating that $18.3 \%$ of all specimens were positive for HPV 18 infection. Another study examining specimens from 417 women attending routine gynecological examinations at three hospitals in Saudi Arabia between April 2010 and December 2011 using PCR technology for detection and an SPF10 DEIA/LiPA25 system for genotyping found $9.8 \%$ prevalence of HPV in Saudi Arabia, with a higher prevalence detected in women who were more than 55 years old or were nonSaudi nationals [18]. Additional studies have been summarized in a publication by Alhamlan et al. [21]. Together these results indicate that studies in individual GCC countries will be required to determine the prevalence of HPV infection before mandating vaccination programs.

\section{HPV and cervical cancer in developing countries}

Worldwide cervical cancer incidence and mortality in 2012 was 528,000 and 266,000, respectively, with

Figure 1. Cervical cancer cases in GCC countries included in the Gulf Center for Cancer Control and Prevention registry over a 14-year span (1998-2012). These data indicate that most cases are discovered at advanced stages.

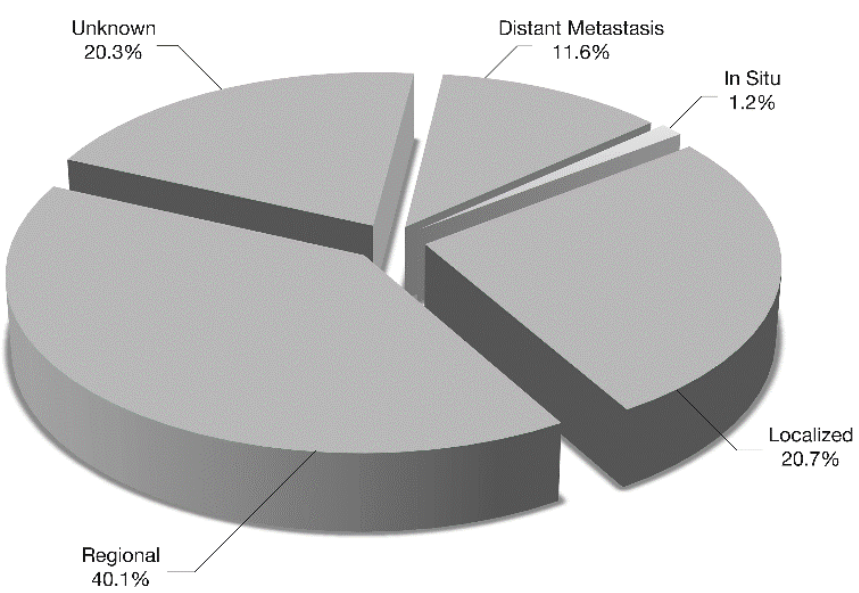


Table 3. Vaccines currently approved by the US Food and Drug Administration.

$\begin{array}{ccc}\text { Trade name } & \text { HPV genotype covered } & \text { Manufacturer } \\ \text { Cervarix } & 16 \text { and } 18 & \text { GlaxoSmithKline } \\ \text { Gardasil } & 6,11,16 \text {, and } 18 & \text { Merck } \\ \text { Gardasil } 9 & 6,11,16,18,31,33,45,52, \text { and } 58 & \text { Merck }\end{array}$

approximately $85 \%$ of cases occurring in developing countries, according to WHO [6]. This same report indicated that the global cervical cancer incidence was highest in Asia (54\%), followed by Africa (19\%), then Central and South America (13\%) and Europe (11\%), and lowest in North America (3\%). The same pattern was detected for mortality rates, with Asian countries, especially the lower-income countries, having the highest mortality rate worldwide $(54 \%)$, followed by African countries (22\%), Central and South America (11\%), Europe (9\%) and, as expected, North America having the lowest mortality rate $(3 \%)$, which is likely attributable to effective early cervical screening, awareness, and vaccination programs.

The cervical cancer burden in developing countries creates a genuine threat to the world because infectious diseases do not recognize borders. Unfortunately, developing countries have neither screening programs nor facilities to implement them, and some of these countries continue to rely on primitive detection techniques. Global efforts should be initiated to provide these countries with the latest technologies to establish successful screening, vaccination, and public education programs to halt sexually transmitted infections, especially HPV [22].

\section{Current diagnostic methods}

Given the high prevalence of HPV as well as the persistence of HPV infections and the long latency for the development of cervical cancer, effective screening for the presence of HPV infection and for cervical cancer at the population level is critical to provide better outcomes for patients worldwide and especially in developing countries. The techniques that have been extensively used for HPV and cervical cancer screening include testing for the presence of HPV or HPVassociated serologic markers (HPV biomarkers) as well as visual inspection, conventional cytology, liquidbased monolayer cytology, or histological examination of the cervix $[23,24]$. These techniques and the advantages and disadvantages of traditional cancer screening tools for cervical cancer have been reviewed in detail by Stack et al. [23]. Briefly, the Pap test is considered the gold standard for cervical cancer screening. After its introduction into standard clinical practice in the $1950 \mathrm{~s}$, cervical cancer decreased by $60 \%$ in women under the age of 55 [25]. HPV infection is primarily diagnosed using molecular biological methods because culturing the viruses are unfeasible and serological methods are insufficiently sensitive (26). Nucleic acid-based screening tools, such as PCR, quantitative reverse transcription PCR, microarray, and next-generation sequencing, are now widely used to facilitate the detection, quantification, and gene expression of HPV viruses [27, 28]. Next-generation sequencing is a high-throughput technique that has been shown to be a useful tool for HPV detection and genotyping [29, 30]. Although high-throughput nextgeneration sequencing is becoming the dominant technology in the discovery and experimental validation of HPV sequences, it may still be too expensive for practical routine use. Therefore, the molecular methods, including DNA-based assays (e.g., PCR, in situ hybridization, reverse line plot, and Hybrid Capture 2) and RNA-based assays (e.g., mRNA gene expression and protein assays) may be most practical in terms of accuracy and speed. Currently, more than five such assays have been approved by the US Food and Drug Administration for HPV detection, including the Digene HC2 HPV DNA Test by Qiagen, the Cobas HPV test by Roche Molecular Diagnostics, and the Cervista HPV HR, the Cervista HPV 16/18, and the Aptima HPV assays by Hologic. Shah et al. has reviewed these assays in detail, describing their detection targets and mechanisms, sample processing, and clinical sensitivity and specificity [23].

\section{Prophylactic HPV vaccine}

In 2009, WHO recommended routine HPV vaccination for girls provided that (1) the goal is to provide prevention of cervical cancer and other HPVrelated diseases, (2) the introduction of a vaccination program is feasible, (3) sustainable financing can be secured, and (4) vaccine strategy cost-effectiveness in the country or region has been considered [31]. Unfortunately, adolescent immunization is relatively rare, with a quarter of world's population underimmunized and vulnerable to a number of preventable diseases, including HPV infection [32]. Although studies have shown that strategies to improve coverage for HPV vaccines result in a significant decrease in the prevalence of HPV by $44 \%$ and genital warts by $33 \%$, controversies regarding HPV vaccinations remain [32]. The US Food and Drug Administration approved HPV 
bivalent (Cervarix, GlaxoSmithKline Biologicals), quadrivalent (Gardasil, Merck \& Co., Inc) and 9-valent (Gardasil, Merck \& Co., Inc) vaccines (Table 3) although whether the first dose should be recommended for children as young as 9 years old remains under debate [33]. Some authorities and governments argue that sexual contact does not exist outside of marriage in some cultures; thus, they do not favor HPV vaccinations for young people. Despite these controversies, however, many people and organizations advocate the use of vaccines, especially in developing countries. However, attempting to implement vaccination programs based on international guidelines may not be feasible or advisable in all countries. In Saudi Arabia, for example, the age at which sexual activity is initiated varies and is supposed to be dependent on marriage. Therefore, it will be important to conduct studies in each nation to determine appropriate vaccination age recommendations. Recent studies have shown that the knowledge and perception of HPV infection as a sexually transmitted disease and the importance of HPV vaccination are low in Saudi Arabia. In one recent survey study, of the 325 patients who visited family medicine clinics at King Faisal Specialist Hospital and Research Centre in Riyadh, only $34.5 \%$ were aware of HPV infection and $27.4 \%$ were aware of its relationship to cervical cancer. The authors found that older and educated individuals were more knowledgeable about HPV infection and vaccination. Although these results are from a hospital-based study, they represent the level of knowledge of HPV infection and vaccination in the kingdom and indicate that awareness campaigns are needed. Other supporting studies have shown a lack of awareness regarding HPV infection, screening, and vaccination among Saudi nationals for not only the general public but also healthcare professionals. Al-darwish et al. conducted a cross-sectional study that provided self-administered questionnaires to 188 medical students. The majority of the students were unaware of the early warning signs, symptoms, and risk factors of cervical cancer. Approximately $55 \%$ of the men and $46.8 \%$ of the women failed to correctly answer the question regarding HPV infection as the cause of cervical cancer. Moreover, $67 \%$ of the medical students were unaware of the availability of HPV vaccines [34]. Another study supporting the claim that the awareness of HPV, cervical cancer, and HPV vaccination is low in Saudi Arabia recruited a random sample of 1023 adolescent girls and women (16-45 years old), who filled out a questionnaire [35]. The researchers found that the majority of the participants $(84.6 \%)$ did not know anything about HPV, 37\% had never heard of a Pap test, and most had never undergone a Pap test [35].

Awareness and health education campaigns are important and cost-effective interventions that should be implemented in developing countries prior to the initiation of screening programs. Large-scale media campaigns offer substantial exposure, increasing public awareness and health education as well as enhancing public perception and response to campaigns to effect greater positive health behavior changes. Mass media campaigns through the routine use of existing media, including social media, television, radio, and newspapers, have been widely used to expose high proportions of large populations to messages. Given the media's recent emphasis on HPV along with the availability of a vaccine, it would be important to gauge the current understanding and awareness of HPV in young men and women. Unfortunately, very few studies have assessed levels of HPV awareness and cervical cancer screening knowledge since the introduction of the HPV vaccine. Embarrassment at even discussing HPV infection as well as ignorance and fear are barriers that remain to be overcome before effective screening and vaccination programs can be successfully initiated. Therefore, the investment by developing countries in well-conducted, long-lasting, and well-funded campaigns to achieve wide population exposure to media messages should be considered as a prerequisite for community-based programs and policies [36,37].

\section{Challenges}

It has been determined beyond any reasonable doubt that high-risk HPVs are etiological agents for cervical carcinogenesis [38]. This leaves clinicians and healthcare providers with the challenging tasks of establishing screening platforms for HPV diagnosis and following up when high-risk HPVs are detected. Scientists also continue to be challenged as they decipher the meaning of a high prevalence of HPV but a low number of cervical cancer cases, especially in areas or countries of concern, and determine the effects of diet, genetic predisposition, and environmental factors on the persistence of HPV infection and its progression to cancer. Indeed, researchers studying cervical cancer rates in 175 countries found that incidence is reflected by country-specific behavioral, health, economic and demographic characteristics. One study conducting regression analyses for 127 low- or medium-developed countries determined that geography and religion are independently associated with high cervical cancer rates. Among behavioral 
measures, high fertility rates, an early age of the mother at the birth of her first child, and high teenage birth rates are significantly associated with high cervical cancer rates. In addition, countries with high cervical cancer rates have fewer doctors per capita, less immunization coverage, more HIV infections, and shorter life expectancies. Moreover, cervical cancer rates tend to be high in countries with less spending on health as well as those having a younger, less educated population [39]. The challenges to both clinicians and scientists are more pronounced in developing countries that do not have established cervical cancer prevention strategies, infrastructure to support vaccination plans, health and awareness education programs, or methods of direct communication with the public. Dissemination of information is necessary to increase public awareness to overcome societal and cultural issues related to sexually transmitted infections. The cultural and religious sensitivities of some countries likely generate avoidance of HPV research and screening programs when there is a dominant belief that HPV infection is associated with unfaithful sexual behavior.

The positive outcomes of cervical cancer screening programs in developed countries may serve as an exemplar for developing countries. There are 19 countries in the International Cancer Screening Network who have established cervical cancer screening programs, including the United States, United Kingdom, Australia, France, Netherlands, and Italy [40]. These countries have extensive experience in cancer prevention, management, and policy that developing countries can adopt. Canada, for example, started screening programs in 1960, followed by Denmark in 1962. Although successful screening and vaccination programs may appear to be initially expensive at the individual level, they are for more costeffective than cancer treatment. In Saudi Arabia, for example, although a Pap test is free for people who have access to public hospitals, it is approximately $\$ 50$ in private clinics, and HPV vaccination is approximately $\$ 400$. Still, these costs are negligible compared with those for treating cervical cancer. According to some studies, the average costs for individuals 6 months after a diagnosis of cervical cancer are $\$ 3,807, \$ 23,187$, $\$ 35,853$, and $\$ 45,028$ for in situ, local, regional, and distant cancers, respectively. The incremental costs of cancer treatment for local and regional cancers are $\$ 13,935$ and $\$ 26,174$, which increase by 12 months to $\$ 15,868$ and $\$ 30,917$, respectively [41]. Thus, in addition to helping to prevent the hardship and adverse effects facing women diagnosed with cervical cancer, screening programs also mitigate the financial burden.

\section{Conclusions}

Cervical cancer and other HPV-related diseases remain global health problems. Although advancement has recently been made in molecular detection and HPV prophylaxis, mortality from cervical cancer is still high in developing countries. Given that HPVs are known etiological agents for cervical cancer, implementation and support of national HPV immunization programs as well as cervical screening would lower the incidence of HPV and its associated diseases, improving the future health of girls and women throughout the world. Nevertheless, the strategy of recommending HPV immunization in developing countries awaits further rigorous evidence at the national level.

\section{Acknowledgements}

We would like to thank King Abdulaziz City for Science and Technology for supporting our research (13-MED2127-20).

\section{References}

1. Scherer WF (1954) Studies on the propagation in vitro of poliomyelitis viruses. VI. Effect on virus yield of cell population, virus inoculum and temperature of incubation. $\mathrm{J}$ Immunol 73: 331-336.

2. Nour NM (2009) Cervical cancer: a preventable death. Rev Obstet Gynecol 2: 240-244.

3. zur Hausen H (2009) Human papillomavirus \& cervical cancer. Indian J Med Res 130: 209.

4. Zheng ZM, Baker CC (2006) Papillomavirus genome structure, expression, and post-transcriptional regulation. Front Biosci 11: 2286-2302.

5. Fernandes JV, Meissner Rde V, Carvalho MG, Fernandes TA, Azevedo PR, de Azevedo JW, de Araujo JM (2011) Human papillomavirus prevalence in women with normal cytology and with cervical cancer in Natal, Brazil. Mol Med Rep 4: 13211326.

6. Jung HS, Rajasekaran N, Ju W, Shin YK (2015) Human papillomavirus: Current and future RNAi therapeutic strategies for cervical cancer. J Clin Med 4: 1126-1155.

7. World Health Organization. (2012) Cervical cancer. Estimated incidence, mortality and prevalence worldwide. Available: http://globocan.iarc.fr/old/FactSheets/cancers/cervix-new.asp. Accessed 20 July 2017.

8. de Martel C, Ferlay J, Franceschi S, Vignat J, Bray F, Forman D, Plummer M (2012) Global burden of cancers attributable to infections in 2008: a review and synthetic analysis. Lancet Oncol 13: 607-615.

9. Walboomers JM, Jacobs MV, Manos MM, Bosch FX, Kummer JA, Shah KV, Snijders PJ, Peto J, Meijer CJ, Munoz N (1999) Human papillomavirus is a necessary cause of invasive cervical cancer worldwide. J Pathol 189: 12-19.

10. Steenbergen RD, de Wilde J, Wilting SM, Brink AA, Snijders PJ, Meijer CJ (2005) HPV-mediated transformation of the anogenital tract. J Clin Virol 32 Suppl 1: 25-33.

11. Cuschieri KS, Beattie G, Hassan S, Robertson K, Cubie H (2005) Assessment of human papillomavirus mRNA detection over time in cervical specimens collected in liquid based cytology medium. J Virol Methods 124: 211-215. 
12. Apgar BS, Zoschnick L, Wright TC Jr (2003) The 2001 Bethesda system terminology. Am Fam Physician 68: 19921998.

13. Narisawa-Saito M, Kiyono T (2007) Basic mechanisms of high-risk human papillomavirus-induced carcinogenesis: roles of E6 and E7 proteins. Cancer Sci 98: 1505-1511.

14. Al-Othman S, Haoudi A, Alhomoud S, Alkhenizan A, Khoja T, Al-Zahrani A (2015) Tackling cancer control in the Gulf Cooperation Council Countries. Lancet Oncol 16: e246-257.

15. Saslow D, Runowicz CD, Solomon D, Moscicki AB, Smith RA, Eyre HJ, Cohen C, American Cancer S (2002) American Cancer Society guideline for the early detection of cervical neoplasia and cancer. CA Cancer J Clin 52: 342-362.

16. Jumaan AO, Ghanem S, Taher J, Braikat M, Al Awaidy S, Dbaibo GS (2013) Prospects and challenges in the introduction of human papillomavirus vaccines in the extended Middle East and North Africa region. Vaccine 31 Suppl 6: 58-64.

17. Al-Ahdal MN, Al-Arnous WK, Bohol MF, Abuzaid SM, Shoukri MM, Elrady KS, Firdous N, Aliyan R, Taseer R, AlHazzani AA, Al-Qahtani AA (2014) Human papillomaviruses in cervical specimens of women residing in Riyadh, Saudi Arabia: a hospital-based study. J Infect Dev Ctries 8: 320-325 doi: $10.3855 /$ jidc. 4220 .

18. AlObaid A, Al-Badawi IA, Al-Kadri H, Gopala K, Kandeil W, Quint W, Al-Aker M, DeAntonio R (2014) Human papillomavirus prevalence and type distribution among women attending routine gynecological examinations in Saudi Arabia. BMC Infect Dis 14: 643 .

19. Alhamlan FS, Khayat HH, Ramisetty-Mikler S, Al-Muammar TA, Tulbah AM, Al-Badawi IA, Kurdi WI, Tulbah MI, Alkhenizan AA, Hussain AN, Ahmed M, Al-Ahdal MN (2016) Sociodemographic characteristics and sexual behavior as risk factors for human papillomavirus infection in Saudi Arabia. Int J Infect Dis 46: 94-99.

20. Al-Muammar T, Al-Ahdal MN, Hassan A, Kessie G, Dela Cruz DM, Mohamed GE (2007) Human papilloma virus-16/18 cervical infection among women attending a family medical clinic in Riyadh. Ann Saudi Med 27: 1-5.

21. Alhamlan FS, Al-Qahtani AA, Al-Ahdal MN (2015) Current studies on human papillomavirus in Saudi Arabia. J Infect Dev Ctries 9: 571-576 doi: 10.3855/jidc.6538.

22. Catarino R, Petignat P, Dongui G, Vassilakos P (2015) Cervical cancer screening in developing countries at a crossroad: Emerging technologies and policy choices. World J Clin Oncol 6: 281-290.

23. Shah SS, Senapati S, Klacsmann F, Miller DL, Johnson JJ, Chang HC, Stack MS (2016) Current technologies and recent developments for screening of HPV-associated cervical and oropharyngeal cancers. Cancers: 8.

24. Stack PS (1997) Pap smears. Postgrad Med 101: 207-214.

25. Sasieni P, Adams J (1999) Effect of screening on cervical cancer mortality in England and Wales: analysis of trends with an age period cohort model. BMJ 318: 1244-1245.

26. Molijn A, Kleter B, Quint W, van Doorn LJ (2005) Molecular diagnosis of human papillomavirus (HPV) infections. J Clin Virol 32 Suppl 1: 43-51.

27. Schiffman M, Wentzensen N, Wacholder S, Kinney W, Gage JC, Castle PE (2011) Human papillomavirus testing in the prevention of cervical cancer. J Natl Cancer Inst 103: 368-383.

28. Silins I, Avall-Lundqvist E, Tadesse A, Jansen KU, Stendahl U, Lenner P, Zumbach K, Pawlita M, Dillner J, Frankendal B (2002) Evaluation of antibodies to human papillomavirus as prognostic markers in cervical cancer patients. Gynecol Oncol 85: $333-338$.
29. Yi X, Zou J, Xu J, Liu T, Liu T, Hua S, Xi F, Nie X, Ye L, Luo Y, Xu L, Du H, Wu R, Yang L, Liu R, Yang B, Wang J, Belinson JL (2014) Development and validation of a new HPV genotyping assay based on next-generation sequencing. Am J Clin Pathol 141: 796-804.

30. Chan PK, Picconi MA, Cheung TH, Giovannelli L, Park JS (2012) Laboratory and clinical aspects of human papillomavirus testing. Crit Rev Clin Lab Sci 49: 117-136.

31. World Health Organization (2009) Human papillomavirus vaccines WHO position paper. Available: http://apps.who.int/iris/bitstream/10665/255353/1/WER9219. pdf. Accessed 11 July 2017.

32. Das JK, Salam RA, Arshad A, Lassi ZS, Bhutta ZA (2016) Systematic review and meta-analysis of interventions to improve access and coverage of adolescent immunizations. J Adolesc Health Suppl 59: 40-48.

33. US Food and Drug Administration (2016) Human Papillomavirus Vaccine. Available: https:/www.fda.gov/BiologicsBloodVaccines/Vaccines/Appr ovedProducts/ucm172678.htm. Accessed 20 July 2017.

34. Al-Darwish AA, Al-Naim AF, Al-Mulhim KS, Al-Otaibi NK, Morsi MS, Aleem AM (2014) Knowledge about cervical cancer early warning signs and symptoms, risk factors and vaccination among students at a medical school in Al-Ahsa, Kingdom of Saudi Arabia. Asian Pac J Cancer Prev 15: 25292532.

35. Gari A AA, Mohammed A, Al-Malki F, Melibari M, AlSheikh M, Kalantan M, Al Qethami N, Futtiny S, Al-Essi S, Al Shomrany Y, Alihiby A (2012) The awareness of the HPV's association with cervical cancer and the HPV vaccine among Saudi females. Life Sci J: 2538-2546.

36. Gerend MA, Magloire ZF (2008) Awareness, knowledge, and beliefs about human papillomavirus in a racially diverse sample of young adults. J Adolesc Health 42: 237-242.

37. Wakefield MA, Loken B, Hornik RC (2010) Use of mass media campaigns to change health behaviour. Lancet 376 : 1261-1271.

38. Bosch FX, Lorincz A, Munoz N, Meijer CJ, Shah KV (2002) The causal relation between human papillomavirus and cervical cancer. J Clin Pathol 55: 244-265.

39. Drain PK, Holmes KK, Hughes JP, Koutsky LA (2002) Determinants of cervical cancer rates in developing countries. Int J Cancer 100: 199-205.

40. National Institutes of health (2012) Cervical cancer screening programs in 19 ICSN countries: organization, policies, and program reach. Available: https://healthcaredelivery.cancer.gov/icsn/cervical/screening.h tml. Accessed 22 July 2017.

41. Subramanian S, Trogdon J, Ekwueme DU, Gardner JG, Whitmire JT, Rao C (2010) Cost of cervical cancer treatment: implications for providing coverage to low-income women under the Medicaid expansion for cancer care. WHI 20: 400405.

\section{Corresponding author}

Mohammed N. Al-Ahdal, PhD

Department of Infection and Immunity

King Faisal Specialist Hospital and Research Centre

PO Box 3354 (MBC-03), Riyadh 11211, Saudi Arabia

Tel: +966114427867

Fax: +966114424519

Email: ahdal@kfshrc.edu.sa

Conflict of interests: No conflict of interests is declared. 